\title{
La correspondencia personal. Una fuente documental para la enseñanza de la historia y la formación del profesorado de Primaria y Secundaria*
}

\author{
Personal Epistolary Correspondence. A Documentary Source for History \\ Teaching and Teacher Training in Primary and Secondary Schools
}

\author{
RAFAEL GUERRERO ELECALDE \\ MigUEL JESÚS LÓPEZ SERRANO
}

\section{Resumen}

La enseñanza de la historia se efectúa desde categorías, debido a que está constituida en departamentos que no benefician su comprensión compleja. Por eso, se plantea la necesidad de ubicar a las personas en el eje del proceso didáctico, ejerciendo como lo que fueron, protagonistas de los aconteceres del pasado. Para ello, se plantea que los futuros docentes trabajen con documentos de carácter personal, especialmente con correspondencia personal. Entre otras cuestiones, la explotación de esta documentación resulta fundamental para el desarrollo del pensamiento histórico, la incentivación de los valores críticos, la construcción del conocimiento y el aprendizaje autónomo.

Palabras clave

Didáctica de la Historia; Fuentes Documentales; Correspondencia; Educación; Metodología

\begin{abstract}
The teaching of history is carried out from categories, because it is constituted in departments that do not benefit its complex understanding. For this reason, it is necessary to place people at the heart of the didactic process, acting as what they were, protagonists of the events of the past. To this end, it is proposed that future teachers work with personal documents, especially with personal correspondence. Among other issues, the use of this documentation is essential for the development of historical thinking, the encouragement of critical values, the construction of knowledge and autonomous learning.
\end{abstract}

\section{Keywords}

Didactics of History; Documentary Sources;

Correspondence; Education; Methodology

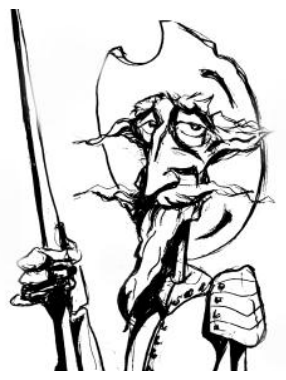

Recibido con pedido de publicación el 15 de enero de 2021

Aceptado para su publicación el 5 de abril de 2021

Versión definitiva recibida el 10 de mayo de 2021

https://doi.org/10.35305/prohistoria.vi36.1527

Rafael Guerrero Elecalde, Universidad de Córdoba, Córdoba, España; e-mail: rgelecalde@uco.es Miguel Jesús López Serrano, Universidad de Córdoba, Córdoba, España; e-mail: mjlopez@uco.es

\footnotetext{
* Agradecemos las sugerencias recibidas por parte de los evaluadores.
}

Esta obra se publica bajo licencia Creative Commons. Atribución-NoComercial-CompartirIgual 4.0 Internacional 


\section{Introducción}

Las diferentes corrientes historiográficas han ido desarrollado su particular procedimiento para la investigación y la enseñanza de la historia. Una de las más transcendentales ha sido la de los Annales, que fue fundada en 1929. Dicha escuela se fue difundiendo de forma progresiva por todo el mundo desde su constitución hasta los años 1980, incorporándose popularmente a los debates científicos más allá de Francia, y del resto de los países europeos, para así convertirse en una de las corrientes historiográficas más importantes del siglo XX (Aguirre Rojas, 1999).

Sus miembros trabajaron, teniendo siempre como base el acontecimiento, por implementar de los temas de la historia más allá de los estudios políticos, bélicos y diplomáticos, predominantes hasta esos momentos, hacia aspectos culturales, científicos o religiosos.

En líneas generales, la metodología de la Escuela de los Annales se define por tres etapas de pensamiento, cada una de ellas con un enfoque distinto, que se encuentran influenciadas por los historiadores más importantes de la época. (Martínez Shaw, 1999): En un primer momento, Lucien Febvre y Marc Bloch se opusieron absolutamente a la visión tradicional de la historia, mientras que en una segunda etapa, Fernand Braudel y Ernst Labrousse la reconfiguraron como una corriente de pensamiento; Por último, sin destacar exponentes claros, se fragmenta el conocimiento historiográfico de sus miembros, y el enfoque socioeconómico de la época se convierte en algo sociocultural (Bourde \& Martin, 1992).

Dicha corriente historiográfica desarrolla una historia que no se interesa por el acontecimiento político y el individuo como protagonistas típicos del trabajo de la Historiografía contemporánea, sino por los procesos, las estructuras sociales $y$, después, por una amplia gama de temas cuyo acercamiento con las herramientas metodológicas de las Ciencias sociales le permitió estudiar. Se trata de una escuela especialmente compleja, en la que, por ejemplo, sus componentes aplicaron técnicas economicistas, serialistas, defendieron una historia de las mentalidades, reflexionaron sobre la temporalidad y las duraciones, para así también anteponer objeciones al simplismo del positivismo (Burguière, 2009).

El historiador en esta corriente se adhiere a un modo de escribir la historia desde el planteamiento de problemas que resolver o preguntas que contestar (Braudel, 1972). En esta línea de trabajo, Pierre Vilar asentó el concepto de historia total, dedicada a una construcción capaz de enlazar los distintos niveles de la actividad social (Hermida, 2006).

Sin embargo, especialmente durante las décadas de 1960 y 1970, las propuestas de la historia cuantitativa o el auge de la historia económica relegaron a los últimos planos de la escena los abordajes cualitativos, situación 
que se reflejaría en la producción de la investigación. Desde entonces, hubo una gran atención a temas económicos, en busca tanto de rigor como de la conceptualización y teorización (Bourde y Martin, 1992; Fernández Clemente, 1995). De este modo se constituyeron, por ejemplo, la "New Economic History" norteamericana y los planteamientos institucionales de la llamada "Escuela de Chicago".

Asimismo, y debido a la gran influencia marxista, se fue uniendo una corriente que incorporó un enfoque con compromiso social y político que, partiendo de presupuestos fundamentados tanto en la historia como en la sociología, fue derivando en planteamientos relacionados con el economicismo y el culturalismo. Buena muestra de ello fueron las publicaciones de los historiadores marxistas británicos M. Dobb, R. Hilton, C. Hill, E. Hobsbawm y E.P. Thompson (Kaye, 1989).

Todos estos planteamientos colaboraron para que en la década siguiente se originara un cambio de paradigma, relacionado con la historia social, que restituyó el protagonismo a las miradas sustentadas en fuentes cualitativas, aumentando el interés por temas antropológicos, a las ideas, a las mentalidades y a las representaciones, para también tener en cuenta otros planteamientos críticos. Así se inauguró la "historia cultural", que situó a hombres y mujeres del pasado en el foco de la observación, analizando a través de sus relaciones y de los conflictos que se originaban. De esta manera, concedió protagonismo a los textos, tanto por su construcción material como por la información que en ellos se alberga, con el deseo de superar la literalidad de sus contenidos y para conocer mejor al sujeto que los redacta (Ríos, 2009; González, 2013).

Desde otras posiciones, Josep Fontana (2002) defendió sobre una realidad incuestionable: "el protagonista de la historia es el hombre en sociedad"; es decir, los hombres y las mujeres en sociedad. Personas que van cimentando los procesos históricos como consecuencia de sus actuaciones y experiencias personales, en un pasado que está vinculado (una historia interconectada), que no se debe compartimentar arbitrariamente con criterios excesivamente rígidos, como las clases sociales, la cronología o la geografía. (Imízcoz, 2017: 1-9).

Entre la variedad de presupuestos metodológicos dirigidos a conseguir la "historia total", hay que reseñar, por su eficacia y garantía, los análisis en clave de red social. Sus planteamientos son los más idóneos porque se trata de una privilegiada vía para entender la dinámica histórica en toda su complejidad, ya que su análisis está profundamente relacionado con la observación de las personas (Imízcoz \& Arroyo, 2011: 98-138). De este modo, se descartan resultados prefijados, observando la historia como el campo y el futuro como lo posible y se aboga por un análisis del pasado desde una perspectiva constructivista. 


\section{Una proposición para un nuevo desafío. La enseñanza de la historia a través de las personas}

Salvo algunos casos aislados, y a pesar de grandes esfuerzos, la metodología que se está estableciendo para la enseñanza de la historia, en los diferentes niveles educativos, continúa reproduciendo pautas y modelos tradicionales. De este modo, se está perpetuando una enseñanza de una historia parcial y "deshumanizada".

Por este hecho, se hace necesario una renovación significativa que se convierta en fundamento para la transformación de las tareas de enseñanzaaprendizaje de los diferentes niveles educativos porque la concepción que el futuro profesorado está construyendo de la historia no es la de un proceso social, sino uno de carácter bélico, que está íntimamente asociado a acontecimientos y datos (Estepa, 2017). Para ello, hay que exigir el compromiso tanto de las autoridades académicas como de los dirigentes políticos, pero también la responsabilidad del propio profesorado de historia, quienes deben esforzarse por vincular su labor docente con las actuales corrientes metodológicas para que, de este modo, las siguientes generaciones de docentes puedan cimentar una concepción crítica y reflexiva de los modelos de la historia (García-Morís, 2016). Y es que esta actualización científica está la clave para emprender una evolución de la actividad educativa a todos los niveles (Parra \& Fuertes, 2019). Con este panorama, también es necesario sensibilizar y comprometer al estudiantado en formación de su futura función en las aulas.

En las propuestas que se plantean en estas páginas, se aboga por enseñar la historia que tome como eje principal al individuo. Los hombres y las mujeres en sociedad son sujetos de una historia que es, al mismo tiempo, política, económica, cultural y religiosa; por lo que, partiendo de ellos, es posible construir un aprendizaje de los contenidos con una percepción conectada de los procesos. Por este motivo, resulta aconsejable colocar a los sujetos históricos en el centro del proceso de enseñanza-aprendizaje de la asignatura de historia, siempre huyendo de los modelos positivistas, donde las personas han sido reducidas a "grandes personajes" en "grandes acontecimientos" (Villalón \& Pagès, 2013).

Este camino es obligatorio transitar para formar al alumnado en pensamiento histórico, instrumento principal para dotarlo de materiales de análisis, de comprensión o de interpretación que le facultarán para entender la historia con autonomía, pudiendo construir su propia representación del pasado y contextualizar o interpretar los hechos pasados a través de la consciencia de la distancia que los separa del presente. El perfeccionamiento del pensamiento histórico tiene que convertirse en la pieza angular que desarrolle el debate, intensifique la argumentación, que favorezca la exposición de reflexiones o hipótesis (Santisteban, 2011; Gómez Carrasco, 2014). 
Seixas y Morton (2013) diseñaron seis dimensiones encaminadas a la consecución de la competencia del pensamiento histórico: la importancia de la relevancia histórica del hecho, la comprensión de la dimensión moral o ética de las interpretaciones históricas, la identificación del cambio y la continuidad, el análisis de las causas y consecuencias de lo acontecido, la toma de perspectiva histórica para comprender el pasado y; por último, el manejo de fuentes primarias como pruebas históricas.

Se trata de una propuesta que apuesta por unas prácticas fundamentadas en procedimientos, y no tanto en cuestiones teóricas, lo que permite un acercamiento al método histórico y al aprendizaje de contenidos de segundo orden, como son aquellos vinculados con la acción propia del historiador (Massip \& Pagès, 2016). Sólo de este modo se conseguirá que el aprendizaje sea significativo, rechazando estructuras rígidas que no responden preguntas, que no elaboran respuestas. Por este motivo, se trata de instituir un diálogo, a través de las fuentes documentales, entre los personajes del pasado y los acontecimientos históricos, permitiendo a quien se acerque a él seguir el hilo de los hechos según se produjeron.

Si nos centramos en las fuentes históricas como herramienta para la enseñanza, con su uso en el aula se pueden alcanzar diversos objetivos especialmente en como futuros docentes entre otras cuestiones:

- Incrementar la comparativa pasado-presente, fomentando el nexo entre las personas del pasado con la propia actualidad.

- Estimular la reflexión y el pensamiento crítico en el alumnado, así como los procesos de construcción individual y colectiva del conocimiento, y se favorecerá el descubrimiento, la investigación, el espíritu emprendedor y la iniciativa personal.

- Fomentar el enfoque interdisciplinar del aprendizaje por competencias con la realización por parte del alumnado de trabajos de investigación y de actividades integradas, que le permita avanzar hacia los resultados de aprendizaje de más de una competencia al mismo tiempo.

- Provocar el conocimiento de la labor archivística: metodología, función social, características y posibilidades para la investigación y la docencia.

\section{Las fuentes históricas personales, un excelente instrumento para la enseñanza de la historia: el ejemplo de la correspondencia epistolar}

Es necesario que las personas estén situadas en un espacio central del proceso de enseñanza-aprendizaje del pasado y, para ello, las fuentes históricas son un recurso didáctico ideal para establecer metodologías activas de aprendizaje de 
los contenidos obligatorios de la asignatura de historia (Prieto, Gómez \& Miralles, 2013; Inarejos, 2017).

Para llevar a cabo este cometido, se pueden visitar los archivos de la localidad o de la provincia, más cercanos, en los que conserven fondos de carácter personal o familiar para, de este modo, realizar la selección de los documentos para utilizar en clase. Esta proximidad podría invitar también a realizar una salida didáctica en un centro archivístico, para que se fuera iniciando en el conocimiento de cómo se conserva la documentación y los modos de clasificación de fuentes.

Sin embargo, en estos momentos, y este hecho va a ir en aumento con el tiempo, existe un amplio acceso a la documentación original digitalizada a través de plataformas de Internet, tanto en los de carácter estatal como en los municipales o privados, nos está otorgando una gran variedad de posibilidades que sin duda ayudará a la renovación de la acción docente y estos recursos deben ser aprovechados para las clases.

Entre la gran variedad de documentación existente, son las fuentes personales las que nos aportan más recursos para llegar a las realidades de los individuos del pasado. Los historiadores han percibido las relaciones personales entre actores a través de fuentes muy diversas: registros parroquiales, protocolos notariales (testamentos, capitulaciones matrimoniales, poderes o escrituras de compraventa), la documentación judicial o documentos autobiográficos (Prieto, Gómez Carrasco, Miralles, 2013).

Sin embargo, la correspondencia personal es la única fuente documental que revela interacciones directas - no mediatizadas institucionalmente entre actores sociales. Hace dos décadas, Antonio Mestre (2000) valoraba los epistolarios como fuente histórica "de primer orden" para el conocimiento del pasado. El autor destacó, en ese momento, el tradicional papel de la carta como herramienta generalizada en la sociedad para incentivar las relaciones entre individuos. En ella se muestra las acciones de las personas y sus relaciones, además de mostrarnos sus intercambios (favores, información, ideas, recursos materiales, influencia) o los valores, ideas o preocupaciones por los que actúan y se vinculan entre sí: enfermedades, inversiones económicas, colocación de los hijos, política matrimonial, pleitos por herencia... Lo que también nos ayuda a romper prejuicios sobre las personas que vivieron en el pasado.

Frente a lecturas de fuentes literarias de perfil histórico, la correspondencia es más clara, eficiente y directa, tratándose, en definitiva, de "una conversación de los hombres cuerdos" (Mestre, 2000).

Las cartas personales exponen los actos de hombres, mujeres y de sus relaciones, tal y como ellas las establecieron y ejercieron. También enseñan sus reciprocidades (favores, información, ideas, recursos materiales, influencia...) valores, ideas o inquietudes que los llevaron a actuar y a relacionarse entre sí, 
como pueden ser miedos o angustias por enfermedades, inversiones económicas, colocación de los hijos, política matrimonial o pleitos por herencia.

Su análisis permite comprender mejor cómo fueron las personas del pasado y así también romper algunos prejuicios sobre ellos (Amor, 2012). El carácter subjetivo de quien escribe el documento convierte a la misiva en un instrumento esencial para conocer la vida cotidiana, social, económica y política, convirtiéndose en una fuente de información para el estudio de las sociedades pasadas "desde dentro" (Castillo, 2016). Por otro lado, un epistolario contiene, normalmente, las misivas que recibe el destinatario de las cartas, pero no las suyas propias. El trabajo será más completo cuando se conservan y se tiene acceso a los copiadores de cartas: borradores de las misivas que se enviaban (Imízcoz \& Arroyo, 2011).

Las identificaciones entre la actualidad y el pasado tienen que ser primordial para erigir lazos entre el aprendizaje de la historia y el estudiantado, tradicionalmente desconfiado con las técnicas de enseñanza de esta materia. Y es que el profesorado tiene que constituir puentes por medio de comparaciones que sirvan para que entiendan, practiquen, estimen $y$, principalmente, valoren a través de la manipulación. Las fuentes personales, y en especial la correspondencia epistolar, son un camino extraordinario para establecer esta interrelación, así como para aplicar una metodología de trabajo desconocida en el ámbito educativo (Beaurepaire \& Taurisson, 2003), particularmente, en el marco de la enseñanza de la historia. Sin embargo, pese a su riqueza y accesibilidad, hasta la fecha, todavía se trabaja de forma superficial e ineficazmente, en contraposición con otras áreas, como Lengua y Literatura, donde se contabilizan algunas propuestas concretas de aula.

\section{Propuestas didácticas con correspondencia. Un ejemplo con cartas de familias vascas del siglo XVIII}

Se suele alegar que la correspondencia personal para la Edad Moderna es una fuente escasa, pero en el caso de las élites no es cierto. Se conservan no pocos epistolarios de gobernantes, intelectuales, comerciantes, eclesiásticos y hombres de letras, tanto en archivos privados como en archivos públicos. Otra cosa es que se hayan trabajado con ellos escasa o superficialmente (Abad, 2016).

Desde hace tiempo, en lo que respecta al siglo XVIII español, se viene publicando trabajos que, desde diversos enfoques, han analizado sobre correspondencia epistolar de diferentes tipos y de diversas características, lo que también ha conllevado la publicación de diversos epistolarios personales completos (Urkia \& Risco, 2005; Vidal-Abarca, Verástegui \& Otazu, 1996; Tellechea, 1987). 
En el siglo XVIII, las cartas eran el principal canal de comunicación entre los individuos que sabían leer y escribir. El trabajo en el aula con correspondencia epistolar, como sucede con cualquier otra fuente documental, debe obligar a los docentes a una preparación de la actividad en tres niveles: antes, durante y después (Chaparro \& Guerrero Elecalde, 2020).

Las actuaciones docentes no se pueden circunscribir exclusivamente a una actuación puntual, sin recorrido anterior o posterior. Además, se considera fundamental, en la medida que sea necesario, la cimentación de coaliciones entre los profesionales de la educación, los historiadores y los técnicos de los archivos para crear asociaciones positivas que cristalicen las actuaciones unidas en propuestas concretas, bien trazadas, objetivas, contextualizadas y asequibles al alumnado.

Entre las principales plataformas, al menos para el mundo hispano, donde se puede encontrar documentación digitalizada y fuentes útiles para estas intenciones se encuentran el Portal de Archivos Españoles (PARES: http://pares.mcu.es/), el repositorio digital de archivos particulares de la Fundación Sancho el Sabio, de Vitoria-Gasteiz (FSS, https://www.sanchoelsabio.eus/fondos-digitales/) y la plataforma Dokuklik (https://dokuklik.euskadi.eus/), puesta en marcha por el Gobierno Vasco (Aguinagalde \& Martín, 1996)., así como de otros repositorios con una relevante utilidad en el ámbito escolar, caso de Europeana (https://www.europeana. eu/portal/es), Archive (https://archive.org/) o la Digital Public Library of America (DPLA: https://dp.la/). En estas plataformas también se encuentra correspondencia personal.

También cabe subrayar que están otros soportes digitales, hechos por profesionales de la historia, que nos brindan materiales suplementarios a las propias fuentes documentales que pueden ser utilizados fácilmente en los contextos educativos. Uno de ellos, a través del cual se pueden trabajar las trayectorias de los individuos que aparecen en la documentación consultada, es el Diccionario Biográfico Español (DBE: http://dbe.rah.es/), con más de cuarenta mil fichas de acceso libre elaboradas por más de dos mil expertos. También, aunque menos conocida, es Fichoz (www.fichoz.org), una iniciativa francoespañola del CNRS. Se trata de un conjunto de bases de datos muy válido a la investigación histórica, que alberga más de cien mil individuos con más de medio millón de acciones. Sin embargo, a pesar de tener esa misma lógica, si bien con ciertas diferencias, cabe reseñar que la información del biografiado no se presenta de manera tradicional, sino a través de un listado cronológico con todas las acciones y relaciones que conllevó, y que mantuvo, durante su vida. El eje fundamental de su construcción está en los "acontecimientos" y no en las personas, razón donde reside su mayor interés (Chaparro y Guerrero, 2019). 
De forma sencilla, en este tipo de repositorios documentales y de plataformas podremos encontrar las herramientas necesarias para introducir las cartas en el aula, plantear y establecer programaciones didácticas adecuadas para enfatizar sobre la importancia de los sujetos históricos en la construcción de las dinámicas del pasado (la Historia desde la percepción de los propios protagonistas) y, de este modo, sustentar la base de la didáctica de la historia en los hombres y mujeres del pasado y no desde los departamentos estancos y estructuras cronológicas en los que se está realizando actualmente y que desvirtúan por completo los procesos históricos.

En los siguientes párrafos, se van a presentar varios ejemplos con personas que vivieron en el siglo XVIII. Siguiendo las pautas que se han explicado anteriormente, se han realizado una pequeña de selección de unas correspondencias de diferentes personas que vivieron en aquella época y son útiles para aprender diferentes contenidos.

Entre todas las posibilidades, nos hemos decantado tomar como ejemplo a diferentes miembros de familias vascas por las temáticas tratadas en las cartas y la accesibilidad (letra legible y clara). Tras una búsqueda sencilla en los repositorios Dokuklik (Badator) y de la Fundación Sancho el Sabio, encontramos las cartas de entre otras muchas, la correspondencia personal del hombre de negocios José Pérez de Vea, el Marqués de los Castillejos, María Vicenta de Barrenechea Castaños y el guipuzcoano Francisco Churruca.

\section{Comercio y comerciantes con Indias en el último tercio del siglo XVIII: Real Compañía de Filipinas}

Nuestra propuesta didáctica está relacionada con el siglo XVIII, más concretamente con la fundación y desarrollo de la Real Compañía de Filipinas (1785), continuadora de la Real Compañía Guipuzcoana de Caracas (1728). La de Filipinas fue la principal compañía privilegiada de comercio por acciones que obtuvo el monopolio comercial para la realización de actividades económicas estables entre Asia y España, a través de la ruta del Cabo de Buena Esperanza, reforzando el papel de estas islas en el entorno asiático.

Su estudio y aprendizaje se puede abordar en el aula a través del análisis de una selección la correspondencia intercambiada entre el comerciante gaditano José Vea-Murguía y Justo Pastor de Asteguieta y Sarralde, antiguo comerciante en Manila, residente en Foronda (Álava), que invierte en los negocios de Filipinas. Para completar el aprendizaje también se pueden analizar las cartas del marqués de los Castillejos al propio Justo Pastor, que son complementarias a las anteriores por los asuntos que tratan. 
Estas cartas se conservan en el fondo Asteguieta, serie Administración de Patrimonio, subserie Correspondencia, de los archivos privados que se conservan en la Fundación Sancho el Sabio (Vitoria-Gasteiz).

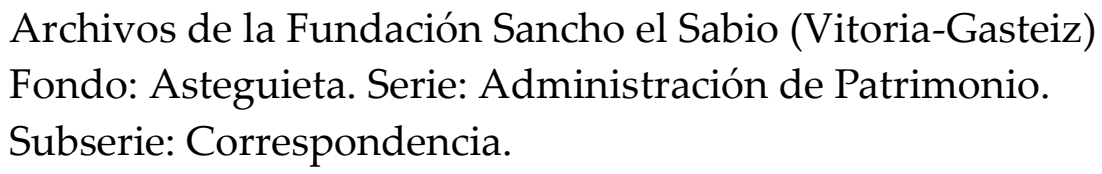

Cartas de José Pérez de Vea a Justo Pastor de Asteguieta Díaz de Sarralde. FSS_A.AS._Asteguieta, C.4, N.2. Fecha inicial y fecha final: 1788-1-8 1794-10-15

Cartas del marqués de los Castillejos a Justo Pastor de Asteguieta Díaz de Sarralde.

FSS_A.AS._Asteguieta, C.3, N.28. Fecha inicial y fecha final:

1775-12-15 - 1791-11-8

Cartas seleccionadas de este fondo:

Desde Cádiz: 22-01-1788; 12-02-1788; 22-02-1788; 11-07-1788; 26-08-1788; $10-$ 03-1789; 19-05-1789; 03-07-1789.

Desde Madrid: 14-11-1793, 27-01-1794; 27-03-1794; 03-04-1794; 19-05-1794; $15-$ 10-1794.

Desde todas ellas, se puede examinar el comercio desde la mirada de estos hombres de negocios: sus problemas, intereses, inversiones en el comercio ultramarino, las actividades económicas que gestionaban, las relaciones de confianza, de amistad y de compromiso mutuo.

Dada la amplitud de los temas, el docente debería hacer un trabajo previo para dirigir correctamente la actividad. De cara a una mejor comprensión de las cartas, se hace necesario ofrecer al alumnado información de los corresponsales (Justo Pastor de Asteguieta, marqués de los Castillejos y José de Vea) para que tengan un conocimiento previo tanto de sus realidades como de familias.

En este sentido, se puede examinar la biografía publicada por la Enciclopedia Auñamendi, su expediente a pasajero a Indias (1774) y la biografía de su hermano José Bernardo de Asteguieta.

A través de la actividad, el alumnado aprenderá por sí mismo y profundizará sobre contenidos relacionados con el mercantilismo español (en 
su derivación de las compañías privilegiadas de comercio) y las políticas desarrolladas por el llamado "absolutismo ilustrado". Asimismo, podrá reflexionar sobre otros temas asociados a los mencionados anteriormente, como "la sociedad estamental" y la "burguesía", en realidad cuestiones controvertidas y que aparecen en los libros de texto.

Para este fin, se pueden plantear actividades para se pregunten sobre $i$ en qué ciudad se producen las ventas de la Compañía? ¿Por qué? ¿Cómo se producen esas ventas? ¿Quiénes compran esos productos? ¿Con qué capitales cuenta una casa de comercio de esta época? ¿Cómo lo consigue? ¿De dónde provienen? ¿Cuáles son las distancias, los tiempos y el conocimiento de estos territorios lejanos por parte de estas familias?

\section{Mujeres en la sociedad ilustrada}

Igualmente, en dichos portales digitales también se albergan fuentes documentales directamente relacionadas con las mujeres, o que hayan sido elaboradas por ellas, y que guardan características propias que venimos planteando. En este sentido, se encuentra la correspondencia de María de Abaria (1792-1796), custodiada en la subsección Vélez de Ulívarri, Serie: X, del Archivo de la Casa de Zavala (Signatura: 250.5); las cartas entre Antonia Ignacia de Ampuero y Pedro María de Ampuero Musaurieta (año 1798), conservada en la sección Familia Ampuero, del Archivo de la Familia Ampuero, (Signatura: FSS, A.F.A., Ampuero, N. 2582) o las de María Vicenta con su sobrino el conde de Hervías (Signatura: FSS, A.U., Manso de Zúñiga, N. 793), que han digitalizado la Fundación Sancho El Sabio, de Vitoria-Gasteiz (España).

En este caso, observamos que se trata de mujeres que nacieron en familias muy ligadas o cercanas al movimiento ilustrado, que saben leer, escribir y contar, incluso expresarse en francés, que muchas de ellas participan en tertulias y escriben sus propios diarios, así como otra literatura.

Una buena muestra la obtenemos con María Vicenta de Barrenechea Castaños, descendiente tanto por rama paterna y materna de importantes familias vizcaínas en las que, además de ocupar importantes cargos a nivel local y provincial, sus miembros estaban desarrollando importantes carreras en el ámbito de la Monarquía en diferentes ámbitos, como el cortesano, la administración, el ejército o el comercio indiano. (Ruiz de Azúa, 1990).

En la correspondencia de María Vicenta con su sobrino el conde de Hervías (FSS, A.U., Manso de Zúñiga, N.793), con fechas extremas entre 1 de noviembre de 1798 y el 5 de marzo de 1820, se pueden contemplar los diferentes asuntos personales, familiares, económicos, ideológicos, políticos, religiosos (...) que preocuparon a esta mujer y a sus allegados; por las características propias 
de esta familia, cuestiones particulares que están íntimamente relacionadas con la política más amplia de la Monarquía.

Junto con estas cartas, si queremos ampliar las actividades y completar los objetivos prediseñados, podremos trabajar con otro tipo de documentación personal como contratos matrimoniales, testamentos, o inventarios de bienes, para comprender el mundo material e inmaterial que rodeaba a estas mujeres. De este modo, en su inventario ("preliminar") de bienes, realizado en el año 1813, (FSS, A.U. Manso de Zúñiga, N.1661) se contemplan cada uno de los artículos que María Vicenta poseía en su casa, estancia por estancia.

Para aprender sobre su papel en la sociedad del siglo XVIII sería interesante presentar cuestiones del tipo: ¿Qué asuntos gestionaban con plena autonomía? ¿Hasta qué punto eran dueñas de su vida? ¿Influían en la política de sus casas? ¿De qué manera? ¿En qué se asemejan o diferencian con las mujeres de ahora?

\section{Educación, carreras al servicio al rey y formación de elites dirigentes del siglo XVIII}

Con la llegada de los Borbones al trono de España, en 1700, se produjo una renovación de las élites gobernantes de la monarquía; familias fieles a la causa Borbónica en la Guerra de Sucesión que fueron reproduciéndose en los espacios de poder a lo largo de la centuria participando activamente en reforma de las diferentes administraciones de la Monarquía, en el ejército, la Armada, los negocios y la Iglesia. Estas familias, como han demostrado las investigaciones recientes formaron parte de los sectores de la sociedad española más modernizantes, convirtiéndose en agentes principales del reformismo ilustrado, en claro contraste con las teorías tradicionales que defendían la existencia de una "revolución burguesa", que terminaría con el Antiguo Régimen y establecería un nuevo orden.

La correspondencia personal de las familias que participaron en ese proceso permite analizar la política de colocación de los vástagos de cada generación por los parientes ya establecidos en la anterior: los mecanismos de colocación y apadrinamiento de hermanos menores, hijos, primos, sobrinos, e incluso sobrinos-nietos; las bases educativas de aquellas carreras y la financiación de los estudios; las trayectorias geográficas y curriculares de los niños por los caminos del parentesco; así como la influencia de que gozaban dichas familias, gracias a su capital relacional, para introducirse en carreras reservadas a las elites y en círculos de poder.

La documentación que se propone es la selección de unas cartas entre Francisco de Churruca y José de Echea, importante comerciante en Cádiz que se conservan, junto con buena parte de la correspondencia personal del primero, 
en el fondo del Archivo de la Casa de Churruca, Condes de Motrico, de los Archivos de Euskadi (Badator): Repositorio documental Dokuklik. Estas misivas están comprendidas cronológicamente entre junio de 1776 hasta marzo de 1777 y hablan del hijo de Francisco, Cosme Damián Churruca (reconocido oficial de la Armada que falleció heroicamente en la batalla de Trafalgar), que está preparando su ingreso en la Real Academia de Guardiamarinas y el viaje desde la casa familiar en Motrico (Guipúzcoa) a Cádiz. Las cartas muestran, entre otras cuestiones, cómo los apoyos personales fueron elementos principales para el desarrollo de las carreras de los hijos de estas familias. En este caso, el vínculo familiar lleva a Francisco de Churruca solicitar a Echea que se ocupe de asistir a su hijo, de 15 años, en sus comienzos en la real academia de la Armada. Entre la ayuda que pide tiene que con la documentación a presentar en la escuela, la administración de su mensualidad, la presentación del chico de los altos mandos de la Armada (Bucarelli o Mazarredo) y la integración en la alta sociedad gaditana.

Para aprender sobre estas cuestiones, el alumnado puede reflexionar entre la relación de los grandes acontecimientos (los que salen en los libros de texto) y el devenir diario de personajes de la historia, tomando como ejemplo el caso que se propone.

\section{Conclusión}

La enseñanza de la historia se produce de manera parcelada e inconexa. Los hombres y las mujeres que viven en sociedad protagonizan una historia única, indisoluble, puesto que la historia es el resultado de procesos políticos, económicos, culturales y religiosos que se producen de manera coetánea y que se encuentran protagonizados, en muchas ocasiones, por las mismas personas.

Los contenidos aparecen estructurados a partir de categorías temáticas, geográficas o temporales que impiden, generalmente, un aprendizaje complejo de la historia y conectar los acontecimientos con aquellos que los protagonizaron o los llevaron a cabo. Asimismo, el enfoque didáctico que, principalmente, se está ejecutando en las aulas no parte de los individuos, sino de los hechos, las instituciones o, incluso, las ideas; contenidos que se priorizan dejando de lado a los protagonistas efectivos.

Por este motivo, se reivindica principios metodológicos que dirijan su foco a las historias de vida a través de la explotación de documentación de archivo. Para ello, nuestra propuesta defiende que el individuo, o el sujeto histórico, debe ser colocado como eje central del proceso de enseñanzaaprendizaje que se produzca un uso intensivo de fuentes históricas de carácter personal o familiar y que, finalmente, se aplique una metodología que priorice la interpretación de la documentación y de los individuos que en ella aparecen actuando. Y es que el estudio de la historia, partiendo de los sujetos históricos, 
ayuda a interrelacionar bidireccionalmente lo que está aconteciendo hoy con lo que ocurrió en el pasado.

Entre todas las fuentes documentales, la correspondencia epistolar resulta la más enriquecedora como recurso didáctico. Y es que la explotación de este tipo de fuentes es clave para el desarrollo del pensamiento histórico y hacia una perspectiva global, así como para la incentivación del pensamiento crítico, la construcción del conocimiento y el aprendizaje autónomo, especialmente en las etapas media y superior de la educación.

Finalmente, hay que destacar que esta reflexión ha buscado contribuir a la capacitación del para el oficio de historiador, adquiriendo competencias sólidas para la docencia para impartir en esta materia.

Córdoba (España), 7 de enero de 2021

\section{Referencias bibliográficas}

Abad, M. (2016). Muy señor mío, dueño y amigo. Lengua epistolar y elite social en la correspondencia privada del sureste español (1760-1805), Tonos digital: Revista de estudios filológicos, (30), 1-34.

Aguinagalde, F. \& Martín, R. (1996). BADATOR: sistema de información sobre la documentación de archivo de Euskadi. En J. Á. García de Cortázar (Coord.), La memoria histórica de Cantabria, (pp. 351-355). Universidad de Cantabria.

Aguirre Rojas, C. (1999). La escuela de los Annales. Ayer hoy, mañana. Montesinos.

Amor, S. (2012). Las vías de análisis de la correspondencia: Las cartas de los Aparici (siglo XVIII). En E. Serrano Martín (Coord.), De la tierra al cielo: Líneas recientes de investigación en historia moderna, 2, (pp. 1037-1054). Fundación Española de Historia Moderna, Institución Fernando el Católico.

Beaurepaire, P. y Taurisson, D. (Dirs.). (2003). Les Ego-documents à l'heure de l'électronique. Nouvelles approches des espaces et réseaux relationnels. Publications de Montpellier III.

Bourde, G.\& Martin, H. (1992). Las Escuelas Históricas. Akal.

Braudel, F. (1972). Personal Testimony, Journal of Modern History, XLIV.

Burguière, A. (2009). La Escuela de los Annales. Una Historia Intelectual. Publicacions de la Universitat de València.

Castillo, A. (2016). El mensaje escrito: la carta como medio de comunicación a lo largo de la historia. En Martínez \& Navarro (Coords.), XXI siglos de comunicación en España. Historia de Correos y Telégrafos, (pp. 13-29). Sociedad Estatal de Correos y Telégrafos S. A. 
Chaparro, Á. \& Guerrero Elecalde, R. (2020). Conectando el pasado. La enseñanza de la historia en Bachillerato a través de la correspondencia epistolar. En F. J. García; C. J. Gómez Carrasco; R. Cózar \& P. Martínez (Coords.), La Historia Moderna en la Enseñanza Secundaria: Contenidos, métodos y representaciones, (pp. 501-512). Universidad de Castilla-La Mancha.

Chaparro, Á. \& Guerrero Elecalde, R. (2019). Actoz: una herramienta para ser historiador en el aula. Íber. Didáctica de las Ciencias Sociales, Geografía e Historia, (97), 76-79.

Estepa, J. (2017). Otra didáctica de la historia para otra escuela. Lección inaugural del curso 2017-2018. Universidad de Huelva.

Fernández Clemente, E. (1995). La historia económica de España en los últimos veinte años (1975-1995). Crónica de una escisión anunciada, Revista de Historia Jerónimo Zurita, 71, 59-94.

Fontana, J. (2002). La historia de los hombres. El siglo XX. Crítica.

García-Morís, R. (2016). Propuesta metodológica para el uso de las fuentes históricas demográficas de la Edad Moderna como recurso didáctico. Revista de Didácticas Específicas, (14), 71-85.

Gómez Carrasco, C. (2014). Pensamiento histórico y contenidos disciplinares en los libros de texto. Un análisis exploratorio de la Edad Moderna en $2^{\mathrm{o}}$ de la ESO. Ensayos. Revista de la Facultad de Educación de Albacete, 29 (1), 131-158.

González, V. (2013). Dejando los restos del naufragio. Fragmentos para una historia cultural Argumentos, 26 (72), 219-237.

Hermida, C. (2006). Pierre Vilar, historiador y maestro de historiadores. Revista Historia y Comunicación Social, 11, 45-60.

Imízcoz, J. \& Bermejo, D. (2016). Grupos familiares y redes sociales en la carrera militar. Los oficiales de origen vasco y navarro en el ejército y la marina, 17001808. Cuadernos de Historia Moderna, 41(2), 497-538.

Imízcoz, J. (2017). La clase política del reformismo borbónico: las redes sociales del cambio. Magallánica. Revista de historia moderna, 4 (7), 10-62.

Imízcoz, J. \& Arroyo, L. (2011). Redes sociales y correspondencia epistolar: del análisis cualitativo de las relaciones personales a la reconstrucción de redes egocentradas. Redes. Revista hispana para el análisis de redes sociales, (21), 98-138.

Inarejos, J. (2017). El uso de fuentes históricas y bases documentales en la formación del profesorado de Educación Infantil y Primaria. Revista Electrónica Interuniversitaria de Formación del Profesorado, 20 (2), 157-166.

Kaye, H. (1989). Los historiadores marxistas británicos. Un análisis introductorio. Universidad de Zaragoza. 
Martínez Shaw, C. (1994). Fernand Braudel: el cenit de la escuela de los Annales. Revista de Occidente, 152, 65-80.

Massip, M. \& Pagès, J. (2016). Humanos frente a humanos: la necesidad de humanizar la historia escolar. En C. R. García; A. Doreste \& B. Mediero (Eds.), Deconstruir la alteridad desde la Didáctica de las Ciencias Sociales: Educar para la ciudadanía global, (pp. 447-457). AUPDCS.

Mestre, A. (2000). La carta, fuente de conocimiento histórico. Revista de Historia Moderna, (18), 13-36.

Nicolás, J. (2016). La carta como abstracción, conocimiento y desencuentro. El caso de G. W. Leibniz. Éndoxa. Series Filosóficas, (38), 255-269.

Pagès, J. (2019). Enseñar historia, educar la temporalidad, formar para el futuro. El futuro del pasado, (10), 19-56.

Parra, D. \& Fuertes, C. (Coords.) (2019). Reinterpretar la tradición, transformar las prácticas: Ciencias Sociales para una educación crítica. Tirant humanidades.

Prieto, J., Gómez Carrasco, C. \& Miralles, P. (2013). El uso de fuentes primarias en el aula y el desarrollo del pensamiento histórico. Una experiencia en Bachillerato. Clío: History and History Teaching, (39), 1-16.

Ríos, M. (2009). De la historia de las mentalidades a la historia cultural: notas sobre el desarrollo de la historiografía en la segunda mitad del siglo XX. Estudios de historia moderna y contemporánea de México. Estud. hist. mod. contemp., 37.

Santisteban, A. (2011). La formación del pensamiento social y el desarrollo de las capacidades para pensar la sociedad. En Santisteban, A. \& Pagès, J. (Eds.), Didáctica del conocimiento del Medio Social y Cultural en la Educación Primaria. Ciencias Sociales para aprender, pensar y actuar, (pp. 85-104). Editorial Síntesis.

Seixas, P. \& Morton, T. (2013). The Big Six Historical Thinking Concepts. Nelson College Indigenous.

Tellechea, J. (1987). La Ilustración vasca. Cartas de Xabier María de Munibe, Conde de Peñaflorida a Pedro Jacinto de Álava. Parlamento Vasco-Eusko Legebiltzarra.

Urkia, J. y Risco, A. (Coords.) (2005). La carta como fuente y como texto: las correspondencias societarias en el siglo XVIII. La Real Sociedad Bascongada de los Amigos del País. Real Sociedad Bascongada de Amigos del País.

Vidal-Abarca, J., Verástegui, F. de y Otazu, A. de. (1996). Fausto de Otazu a Iñigo Ortés de Velasco: cartas 1834-1841 (2 vols.). Diputación Foral de Álava.

Villalón, G. \& Pagès, J. (2013). ¿Quién protagoniza y cómo la historia escolar? La enseñanza de la historia de los otros y de las otras en los textos de estudio de Historia de Chile de educación primaria. Clío \& Asociados, (17), 119-136. 